\title{
Editorial
}

\section{The Breast Cancer Sentinel Node Dilemma: What to do with Isolated Tumor Cells and Micrometastases?}

\author{
Vivianne C.G. Tjan-Heijnen \\ Maastricht University Medical Centre, Division of Medical Oncology, Department of Internal Medicine, P.O. Box \\ 5800, 6202 AZ Maastricht, The Netherlands \\ Tel.: +31 43 3877025; Fax: +31 43 3876281; E-mail:vcg.tjan.heijnen@mumc.nl
}

Keywords: Sentinel node, isolated tumor cells, micrometastases, axillary therapy, systemic therapy, breast cancer

This issue of Breast Disease is dedicated to the sentinel node $(\mathrm{SN})$ procedure in breast cancer, and more specifically on how to treat patients in whom isolated tumor cells or micrometastases are detected.

Introduction of population-based breast cancer screening and an increased awareness in the general population regarding breast lumps has resulted in a shift towards clinically node-negative breast cancer stages. As a result, nowadays, around $60 \%$ of breast cancer patients have pathologically node-negative disease [1]. The SN procedure is based on the premise that if the first node the breast tissue drains into is clean, the remaining axillary lymph nodes are likely not involved, with no need for removal. Therefore, the introduction of the SN procedure during the nineties of the previous decade was a way to reduce axillary over treatment in many patients, thereby reducing morbidity such as lymph edema and shoulder dysfunction. However, concerns regarding misinterpretation of SN status and axillary recurrences led to an intensified $\mathrm{SN}$ pathology protocol, which partly counterbalanced the impact of the SN procedure: of patients who are eligible for the SN procedure, on average $10 \%$ have $\mathrm{SN}$ isolated tumor cells, and $10 \%$ have SN micrometastases [2], which would largely have remained undetected if only examined by routine $\mathrm{H} \& \mathrm{E}$ lymph node histology. If such patients would all undergo axillary surgery the impact of the SN procedure in sparing axillary over treatment would be substantially lower as was presumed before. Indeed, we have shown that in the initial years of the SN procedure, where all patients with isolated tumor cells underwent axillary lymph node dissection, a triple intensive SN pathology protocol led to more than twice as many surgical procedures compared to hospitals using a 'standard' SN pathology protocol [3].

It is noticed that till the year 2002, metastases with a diameter of $2 \mathrm{~mm}$ or less were classified as pN1a, without distinction between isolated tumor cells and micrometastases. At that time, most of the patients with minimal nodal involvement were treated as if they were node-positive, including the use of adjuvant systemic therapy. Since 2002, however, due to the implementation of the SN procedure with increasing detection frequency of small nodal metastases and because of doubt about the prognostic relevance of isolated tumor cells, the Cancer Staging Manual of the American Joint Committee on Cancer (6th edition) distinguished between isolated tumor cells ( $\mathrm{pNO}(\mathrm{i}+), \leqslant 0.2 \mathrm{~mm}$ ) and micrometastases ( $\mathrm{pN} 1 \mathrm{mi}, 0.2-2.0 \mathrm{~mm}$ ). In the recently updated 7th edition, the definition of micrometastases has been somewhat widened to include nodal involvement "larger than $0.2 \mathrm{~mm}$ and/or more than 200 cells, 
but none larger than $2.0 \mathrm{~mm}$ ", which prevents large areas of lymph node involvement to be categorized as pNO(i+), and would likely improve reproducibility of staging [4]. These and other pathology issues are addressed by Van Diest and colleagues in their contribution to this Breast Disease issue. They provide an up to date discussion on the virtues and flaws of different methods to find SN metastases, and provide recommendations on the optimal pathology protocol for breast cancer SNs and the best way to classify small nodal metastases.

ASCO guidelines recommend no additional axillary treatment in patients with node-negative disease including those with isolated tumor cells [5]. In patients with a negative $\mathrm{SN}$, many studies have shown that omitting axillary treatment is safe, with an axillary recurrence rate of about $1 \%$ after 5 -years follow-up, in line with validation and randomized studies showing less than $5 \%$ positive non-SNs in SN-negative patients [6]. With increasing SN metastases size the incidence of non$\mathrm{SN}$ involvement rises: $12.3 \%$ with $\mathrm{SN}$ isolated tumor cells [7], 20\% with SN micrometastases [8] and 30\% with SN macrometastases [2]. If left untreated, not all these non-SN metastases, will grow out to axillary recurrences amongst others because of use of adjuvant systemic therapy. However, non-SN involvement rates suggest that the risk of axillary recurrence will be higher for SN positive patients compared with SN negative patients if the axilla is left untreated.

Recently, attention was drawn to the fact that there is a trend towards omitting axillary lymph node dissection in patients with SN micrometastases [9]. In selected patients with a positive SN not treated with completion axillary lymph node dissection, the incidence of axillary recurrence seems low in most studies reported so far. However, in the Dutch MIRROR cohort study it was observed that, after correcting for tumor characteristics and adjuvant systemic therapy, the rate for axillary recurrence in the 141 patients with SN micrometastases who had not undergone axillary treatment was $5.6 \%$, resulting in a hazard ratio for regional recurrence of 4.39 , compared to women whose axilla was treated [10]. Similar results were seen in a series of the Memorial Sloan Kettering Cancer Centre, with axillary recurrences in $2.0 \%$ of SN positive / no axillary surgery patients at a median follow-up of 23 months [11]. Of importance, the highest axillary recurrence rate $(5 \%)$ was seen in the patients whom SNs were positive on routine $\mathrm{H} \& \mathrm{E}$. In this issue of Breast Disease Erb et al. provide an overview on studies concerning axillary recurrence rates following a pos- itive SN. They conclude that for the present, axillary lymph node dissection for SN micrometastasis is still to be recommended in most patients. However, they also stress that in selected patients, after weighing the risks and the benefits of additional axillary treatment, it may be decided in a multi-disciplinary team setting that additional axillary treatment could be omitted.

Recht addresses the question whether radiotherapy can replace axillary dissection for patients with positive SNs. The European Organization for Research and Treatment of Cancer trial 10981, "After Mapping of the Axilla: Radiotherapy or Surgery?" (AMAROS), randomly allocates patients with positive sentinel nodes with primary tumors measuring $0.5-3 \mathrm{~cm}$ to receive either completion axillary dissection or axillary radiotherapy. Results from this trial will shed more light on the effectiveness and safety of axillary radiotherapy versus axillary surgery. In most published series, information why patients did not undergo axillary dissection, why a particular radiotherapy technique was selected, and information regarding the use of systemic therapy were not usually reported. Many patients in these studies were at low risk of having non-SN involvement. Recht concludes that it is not yet clear to what extent radiotherapy can replace axillary dissection after a positive $\mathrm{SN}$ : "Such treatment is likely to be very safe, but there are few data on its long-term effectiveness for different patient subgroups".

So, it is of pivotal importance to estimate non-SN involvement for the individual patient. In this issue of Breast Disease, Pesce et al. provide an overview on published studies regarding prediction of non-SN involvement. These studies show that not only the size of SN involvement but also primary tumor characteristics play a role, such as primary tumor size and presence or absence of lymphovascular invasion. Unfortunately, however, none of these studies have reliably identified a patient population at sufficiently low risk for axillary nodal metastases to avoid axillary treatment. And, as pointed out by the authors, survival benefit of axillary treatment was not (yet) taken into account. The authors conclude that, while awaiting the data from current clinical trials to mature, we must inform our patients about the risks, benefits and imponderables of treating potential axillary metastases and incorporate their viewpoint into a final decision.

Another major question is whether the presence of isolated tumor cells or micrometastases is associated with overall breast cancer outcome. Axillary lymph node status is the most important prognostic factor in breast cancer. But, is this also true for minimal nodal 
involvement? If so, then it seems logical that these patients should be considered for adjuvant systemic therapy. In this issue, the available evidence regarding breast cancer prognosis is summarized. It is shown that the presence of isolated tumor cells or micrometastases is associated with a hazard ratio of about 1.50 (ranging from 1.35 to 1.89 ) for disease-events compared to nodenegative disease, both in studies before and after the introduction of the $\mathrm{SN}$ procedure, although not always using multivariate analysis. The MIRROR study, the largest SN study including nearly 2000 patients with isolated tumor cells or micrometastases, reported a hazard ratio for disease-events of 1.50 (95\% CI 1.15-1.94) for isolated tumor cells and $1.56(95 \%$ CI $1.15-2.13)$ for micrometastases, multivariately corrected for age and tumor characteristics in patients who had not received systemic therapy [12]. Based on the evidence from this overview, it is recommended to multiply the risk of recurrence based on other characteristics such as tumor size, histological grade, absence of estrogen receptor, the HER2 status, age and presence of co-morbidity by 1.50 for patients with isolated tumor cells or micrometastases to decide whether or not to administer adjuvant systemic therapy to an individual patient.

In conclusion, an increasing amount of evidence is becoming available on nodal isolated tumor cells and micrometastases since the introduction of the SN procedure. Data on a number of randomized studies are expected in the next few years. To this end, this issue of Breast Disease provides an overview of current evidence that may be supportive in making the best decision in current patient care.

\section{References}

[1] V.K.Y. Ho, M. van der Heiden-van der Loo, E.J.Th. Rutgers et al., Implementation of Sentinel Node Biopsy in Breast Cancer in The Netherlands, Eur J Cancer 44 (2008), 683-691.
[2] M.J. Bolster, P.G. Peer, P. Bult et al., Risk factors for nonsentinel lymph node metastases in patients with breast cancer. The outcome of a multi-institutional study, Ann Surg Oncol 14 (2007), 181-189.

[3] M.J. Bolster, P. Bult, F.B.J.M. Thunnissen et al., Differences in sentinel lymph node pathology protocols lead to differences in surgical strategy in breast cancer patients, Ann Surg Oncol 13 (2006), 1466-1473.

[4] S.B. Edge, D.R. Byrd, C.C. Compton et al., AJCC Cancer Staging Handbook, from the: AJCC Cancer Staging Manual, Springer, New York, 2009.

[5] G.H. Lyman, A.E. Giuliano, M.R. Somerfield et al., American Society of Clinical Oncology guideline recommendations for sentinel lymph node biopsy in early-stage breast cancer, J Clin Oncol 23 (2005), 7703-7720.

[6] U. Veronesi, G. Paganelli, G. Viale et al., Sentinel-lymph-node biopsy as a staging procedure in breast cancer: update of a randomised controlled study, Lancet Oncol 7 (2006), 983-990.

[7] C.H.M. van Deurzen, M. de Boer, E.M. Monninkhof et al., Non-sentinel lymph node metastases associated with isolated breast cancer cells in the sentinel node, J Natl Cancer Instit 100 (2008), 1574-1580.

[8] G. Cserni, D. Gregori, F. Merletti et al., Meta-analysis of non-sentinel node metastases associated with micrometastatic sentinel nodes in breast cancer, Br J Surg 91 (2004), 12451252.

[9] N. Wasif, X. Ye and A.E. Giuliano, Survey of ASCO members on management of sentinel node micrometastases in breast cancer: variation in treatment recommendations according to specialty, Ann Surg Oncol 16 (2009), 2442-2449.

[10] V.C.G. Tjan-Heijnen, M.J. Pepels, M. de Boer et al., Impact of omission of completion axillary lymph node dissection (cALND) or axillary radiotherapy (ax RT) in breast cancer patients with micrometastases $(\mathrm{pN} 1 \mathrm{mi})$ or isolated tumor cells (pNO[i+]) in the sentinel lymph node $(\mathrm{SN})$ : Results from the MIRROR study, Annual Meeting of the American Society of Clinical Oncology, Orlando (2009), \#CRA506.

[11] J. Park, J.V. Fey, A.M. Naik et al., A declining rate of completion axillary dissection in sentinel lymph node-positive breast cancer patients is associated with the use of a multivariate nomogram, Ann Surg 245 (2007), 462-468.

[12] M. de Boer, C.H. van Deurzen, J.A. van Dijck et al., Micrometastases or isolated tumor cells and the outcome of breast cancer. New Engl J Med 361 (2009), 653-663.

[13] S.L. Chen, M.B.A. Francesca and M. Hoehne, The prognostic significance of micrometastases in breast cancer: A SEER population-based analysis, Ann Surg Onc 14 (2007), 33783384. 